\title{
Studies in a co-infection murine model of Plasmodium chabaudi chabaudi and Leishmania infantum: interferon- $\gamma$ and interleukin-4 mRNA expression
}

\author{
Cláudia S Marques, Nuno Rolão, Sónia Centeno-Lima*, Hélder Lousada, Carla Maia, \\ Lenea Campino, Virgílio E do Rosário*, Henrique Silveira*/+
}

\author{
Unidade de Leishmanioses *Unidade de Malária, Centro de Malária e Doenças Tropicais, Instituto de Higiene e Medicina \\ Tropical, Universidade Nova de Lisboa, Rua da Junqueira 96, 1349-008 Lisboa, Portugal
}

This work aimed to study the $T$ helper type $1 / 2\left(T h_{1} / T h_{2}\right)$ cytokine profile in a co-infection murine model of Plasmodium chabaudi chabaudi and Leishmania infantum. Expression of interferon-gamma (IFN- $\gamma$ ) and interleukin4 (IL-4) was analyzed, in spleen and liver of C57BL/6 mice, by reverse transcriptase-polymerase chain reaction.

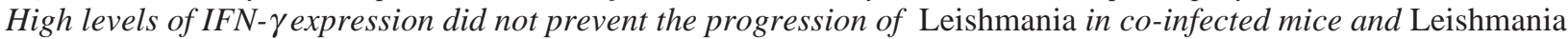
infection did not interfere with the $T h_{l} / T h_{2}$ switch necessary for Plasmodium control. The presence of IL-4 at day 28 in co-infected mice, essential for Plasmodium elimination, was probably a key factor on the exacerbation of the Leishmania infection.

Key words: co-infection - Plasmodium - Leishmania

Malaria and leishmaniasis are currently two of the major causes of mortality and morbility among human parasitic infections, with an enormous social and economic impact. Human cases of concomitant malaria, Plasmodium falciparum and leishmaniasis, Leishmania donovani have been reported (Nandy et al. 1995, Sah et al. 2002).

In 1980s, Coleman and his colleagues developed several studies of interactions betweeen $L$. mexicana and $P$. yoellii in the murine model, that demonstrated an elevation of severity and susceptibility to the diseases during co-infection. However, they did not investigate the immunological mechanisms involved (Coleman et al. 1988a, b, c). The $P$. chabaudi chabaudi/L. infantum murine model could reveal parasite-specific immune responses and mechanisms of regulation of T-cell subsets during a concomitant infection. Regulation of immune response by $\mathrm{CD}^{+} \mathrm{T}$ helper (Th) cells and cytokine products have in fact been shown to be essential during both Plasmodium and Leishmania infections.

P. c. chabaudi strain AS infection, in C57BL/6 mice strain, induces a sequential immune response by the two major subsets of CD4+ Th cells (Langhorne 1989). The acute primary parasitaemia, with a peak level around day 7 , is resolved by the $\mathrm{Th}_{1}$ type response and related cytokines, mainly interferon-gamma (IFN- $\gamma$ ) (Langhorne 1989, Zhong \& Stevenson 2000). The control and clearance of chronic subpatent blood infection during the second phase of infection are related with the $\mathrm{Th}_{2}$ pattern of cytokine production, namely interleukin-4 (IL-4), that is crucial for B cells maturation and subsequent antibody release (Langhorne 1989, Taylor-Robinson \& Phillips 1994).

+Corresponding author. E-mail: hsilveira@ihmt.unl.pt Received 12 August 2005

Accepted 23 November 2005
The murine malaria $P$. $c$. chabaudi is a recognized model for analyzing the acquired immunity to the asexual erythrocytic stages of malaria parasites especially for $P$. falciparum infections of humans (reviewed by TaylorRobinson 1998).

In murine models of leishmaniasis, it is widely assumed that resistance to the disease is correlated with the production of IFN- $\gamma$ by $\mathrm{Th}_{1}$ cells, while susceptibility is associated with $\mathrm{Th}_{2}$ response, resulting in IL-4 production (Heinzel et al. 1989, Gumy et al. 2004), although in visceral leishmaniasis this clear-cut role of $\mathrm{Th}_{1} / \mathrm{Th}_{2}$ is less evident (Honoré et al. 1998).

The main goals of this study were to evaluate whether the initial immune responses to $P$. $c$. chabaudi could be blocked, when mice were co-infected by L. infantum, thus preventing the $\mathrm{Th}_{1} / \mathrm{Th}_{2}$ switch, and to investigate if resistance or susceptibility to L. infantum could be modulated by a previous activation of $\mathrm{Th}_{1}$ cells. In order to clarify these questions, we analysed mRNA expression of IFN- $\gamma$ and IL-4, both in spleen and liver of co-infected C57BL/6 mice, by semi-quantitative reverse transcriptase-polymerase chain reaction (RT-PCR).

\section{MATERIALS AND METHODS}

C57BL/6 female mice, 5 to 8 weeks old were purchased from Harlam Interfauna Ibérica SL (Barcelona, Spain) and kept in the IHMT animal facilities, according to the European Union requirements (86/609/CEE) recognized in Portuguese law (DR DL129/92 and Portaria 1005/92). P. c. chabaudi AS was obtained from the Institute of Cell, Animal and Population Biology, University of Edinburgh and maintained by continuous blood passage. L. infantum MON-1 amastigotes were isolated from spleen and liver of infected golden hamsters (Mesocricetus auratus), according to Cenini et al. (1989).

Mice were divided into the following groups: (i) intraperatonial inoculation with isotonic saline solution, (ii) intraperatonial inoculation with $10^{6}$ P. c. chabaudi AS 
parasitised red blood cells $\left(\mathrm{pRBC}_{\mathrm{c}}\right.$ ), (iii) intraperatonial inoculation with $10^{6} \mathrm{~L}$. infantum (MON-1) amastigotes, and (iv) intraperitonial inoculation of $10^{6} P$. c. chabaudi $\mathrm{AS} \mathrm{pRBC}_{\mathrm{s}}$ and seven days later with $10^{6} \mathrm{~L}$. infantum (MON1) amastigotes.

Three mice of each group were sacrificed by cervical dislocation and fragments of spleen and liver were collected at days 0 (P. c. chabaudi inoculation day), 7 ( $L$. infantum inoculation day), 14, 28, 35, and 56 .

DNA extraction from sample tissue (spleen and liver) was carried out according to Boom et al. (1990) and PCR amplification for the diagnosis of infection caused by $L$. infantum was performed using primers based on a complete DNA sequence of $L$. infantum kinetoplast minicircle (Cortes et al. 2004).

L. infantum load was monitored by determination of parasite density in spleen and liver of groups 3 and 4, using the limiting dilution method (Buffet et al. 1995).

$P$. c. chabaudi parasitaemia was monitored and determined during the course of infection of groups 2 and 4 by optical microscopic observation of thin blood smears made from mice tail snips and stained with Giemsa.

Fragments of spleen and liver collected for RNA extraction were stored in liquid nitrogen until use. Extraction of total RNA was performed with TRIzol ${ }^{\circledR}$ reagent (Invitrogen-Life Technologies, New York, US), according to the manufacturer's protocol. Synthesis and amplification of cDNA were performed as described by CentenoLima et al. (2002). Specific primers for murine IFN- $\gamma$, IL-4 and hypoxanthine-guanine phosphoribosyl transferase (HPRT) as an internal standard were used (Centeno-Lima et al. 2002).

After electrophoresis on 1\% agarose gel, DNA was transferred to a positively charged nylon membrane by common blotting procedures. Blots were hybridised with a specific digoxigenin (DIG) labelled oligoprobe for each cytokine and HPRT as previously described by CentenoLima et al. (2002). The oligonucleotide probe-labelling protocol was performed according to the manufacturer's protocol (Roche Applied Science). Hybridization chemiluminescent signals were quantified and converted to optical density units (OD) using Phoretix 1D plus software (Phoretix International). Results were normalized for the relative quantity of cytokine mRNA (arbitrary units) by dividing the OD of the cytokine by the corresponding OD of HPRT, for each sample. Data analysis was carried out using Statistical Package for Social Sciences 11.0 Program $\left(\mathrm{SPSS}^{\circledR}\right)$. The non-parametric Mann-Whitney rank sum test was used to assess statistical significance for values of $\mathrm{P}<0.05$. The results of the following groups were statistically compared at days $14,28,35$, and 56 after $P$. $c$. chabaudi infection: group 2 vs group 4 and group 3 vs group 4.

\section{RESULTS}

Mice infected with P. c. chabaudi alone (group 2) showed an expected peak of parasitaemia at day $7(37 \%)$ that was followed by a drastic reduction of parasitaemia. Two recrudescences were noted at days 16 and 30 before total eradication of blood parasites, verified by optical microscopy observation (Fig. 1).

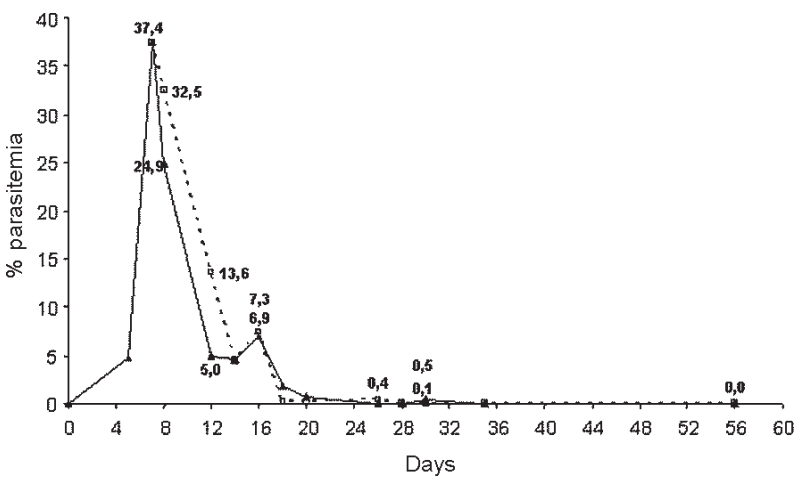

Fig. 1: course of blood stage Plasmodium chabaudi chabaudi infection in $\mathrm{C} 57 \mathrm{BL} / 6$ mice infected with $10^{6} P$. c. chabaudi parasitized red blood cells (pRBC) (full line) and in C57BL/6 mice coinfected with $10^{6}$ P. c. chabaudi pRBC's and 7 days later with $10^{6}$ Leishmania infantum amastigotes (dotted line). Parasitaemia was determined after observation of thin blood smears stained with Giemsa and by counting total and infected red blood cells in five microscopic fields. The total number of red blood cells was equal or superior to 2000. Data represent the geometric mean of 3 mice per group, from the most representative experiment.

All cultures from the spleen and liver samples, collected in mice inoculated with $L$. infantum only (group 3 ) were negative throughout the experiment, however the presence of Leishmania DNA was confirmed in both organs by PCR.

The course of blood stage $P$. c. chabaudi infection in co-infected mice (group 4) was similar to what was described for group 2, though mice from group 4 reached higher levels of parasitaemia until day 14, exhibiting recrudescence at days 16, 26, and 30 (Fig. 1).

Spleen from co-infected mice showed positive promastigote cultures at day 56 , with a parasite density mean value of $8 \times 10^{4}$ parasites per $g$ of organ, as determined by the limiting dilution method. The high Leishmania parasite density observed at day 56 was accompanied by an evident splenomegaly on all three mice.

The IFN- $\gamma$ expression in the spleen and liver of mice infected with P. c. chabaudi only, reached a peak at day 7 that coincided with peak of parasitaemia. The expression level of IFN- $\gamma$ decreased afterwards (Fig. 2a, b).

Co-infected mice showed elevated IFN- $\gamma$ expression at day 56, both in the spleen and liver, and significant differences were noted in the liver between this group and mice infected P. c. chabaudi only (Fig. 2a, b).

In mice infected with $L$. infantum only the IFN- $\gamma$ expression levels were generally lower than in co-infected mice and in spleen, IFN- $\gamma$ expression was only detectable at day 28. Significant differences between group 3 and group 4 were observed in the liver at days 14 and 56 (Fig. $2 \mathrm{a}, \mathrm{b})$.

The IL-4 gene expression was detected in the spleen of groups 2 and 4, with a peak at day 28 . At this day, the IL-4 expression levels were significantly higher in co-infected mice than in Leishmania infected mice (Fig. 2c). This cytokine was not detected in the liver of mice from any group (data not showed). 

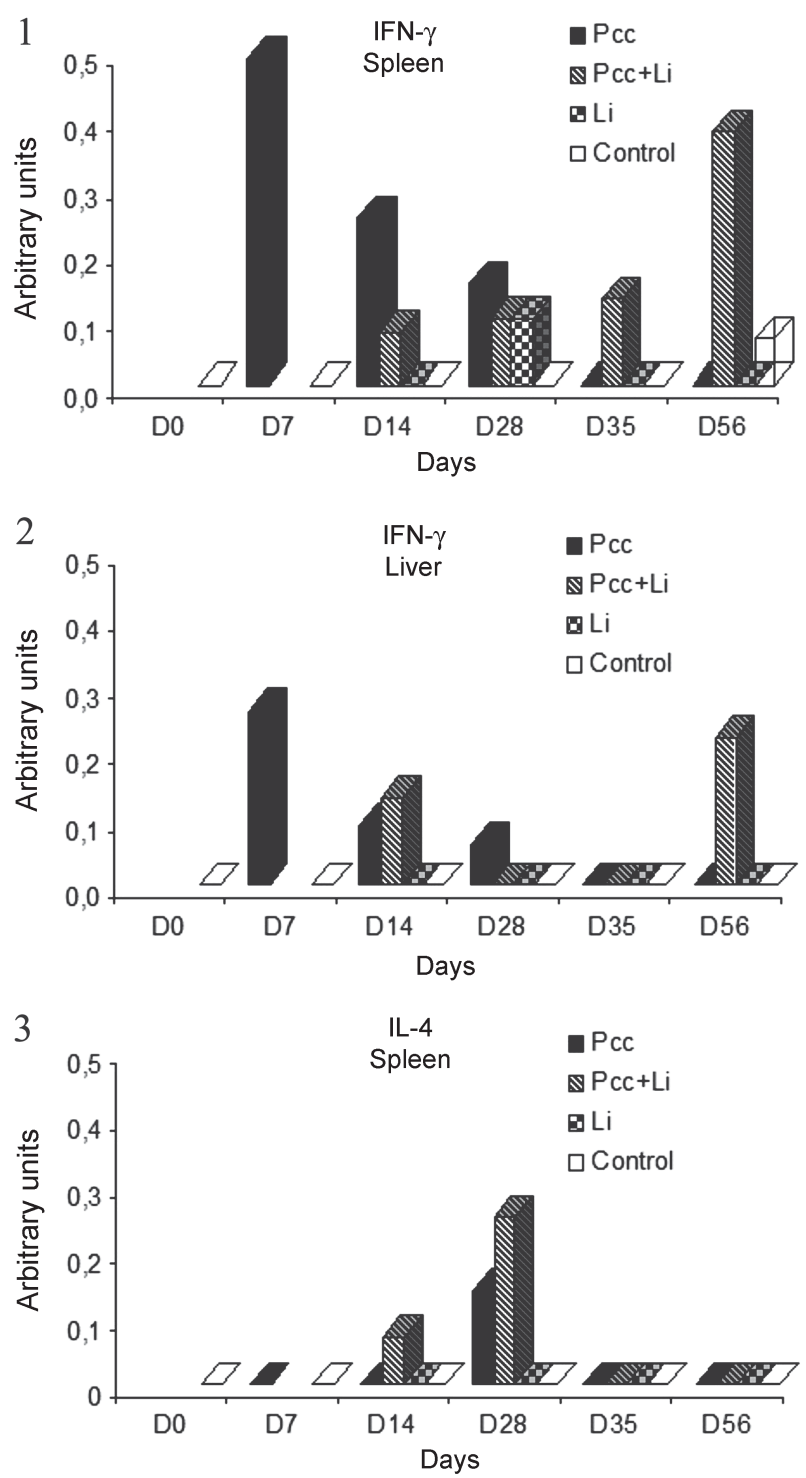

Fig. 2: interferon-gamma (IFN- $\gamma$ ) and interleukin-4 (IL-4) relative gene expression in liver and spleen of C57BL6 mice during Plasmodium chabaudi chabaudi and/or Leishmania infantum infections. Co-infected mice were inoculated at day 0 with $P$. c. chabaudi and at day 7 with $L$. infantum. Cytokine reverse transcriptasepolymerase chain reaction data was normalized by dividing the OD of the cytokine by the corresponding OD of the constitute gene HPRT (arbitrary units). Data represent median of 3 mice per group, from the most representative experiment. 1 and 3 - spleen; 2 liver, Pcc - P. c. chabaudi infected mice (group 2); Pcc+Li - P. c. chabaudi and L. infantum infected mice (group 4); Li - L. infantum infected mice (group 3); Control - mice inoculated with isotonic saline solution (group 1).

\section{DISCUSSION}

Despite the existence of overlapping endemic areas, scarce information is known on concomitant malaria and leishmaniasis and no references are available regarding cytokine analysis in mixed infections involving Plasmodium and Leishmania. In the present study, the analysis of cytokine mRNA expression in spleen and liver cells during the early phase of erythrocytic $P$. c. chabaudi infection, confirmed that in C57BL/6, there is a $\mathrm{Th}_{1}$ inflam- matory response, associated with high expression of IFN$\gamma$ by day 7 post-infection (peak of parasitaemia) in order to control parasitaemia (Langhorne 1989, Zhong \& Stevenson 2000). In parallel, our results confirmed a progressive elevation of IL-4 expression, a Th ${ }_{2}$ cytokine characteristic of the chronic stage of $P$. c . chabaudi infection, responsible for $\mathrm{B}$ cells maturation and antibody release, which are necessary for recrudescence control and total elimination of blood parasites (Langhorne 1989, TaylorRobinson \& Phillips 1994). When all Plasmodium parasites are eradicated from the blood (around day 35), the IL-4 expression becomes undetectable, both in plasmodium-only and co-infected mice.

Although the course of parasitaemia was similar in mice infected only with $P$. c. chabaudi and in co-infected mice, the IFN- $\gamma$ expression level seemed to persist from day 14 onwards in the co-infected group, mainly in the spleen, and then markedly increased at day 56. This sustained IFN- $\gamma$ expression may be related to the fact that on the day of Leishmania inoculation, a $\mathrm{Th}_{1}$ immune response had already been established as a result of the Plasmodium infection. Although some authors point out to the importance of IFN- $\gamma$ in the resolution of leishmaniasis infection (Kaye et al. 1991, Carvalho et al. 1994), the IFN- $\gamma$ expression levels detected at day 7, when Leishmania parasites were inoculated, apparently did not restrain the progression of $L$. infantum infection in co-infected mice. According to Melby et al. (2001), syrian hamsters infected with $L$. donovani are not able to control parasite replication despite the high levels of $\mathrm{Th}_{1}$ cytokine expression, namely IFN- $\gamma$, as observed in this study. In fact, co-infected mice presented an evident splenomegaly on day 56 , which is a typical clinical symptom of visceral leishmaniasis, and positive cultures with high values of parasite density were observed. In addition, there was no detectable erythrocytic Plasmodium parasites. Concomitant Plasmodium infection seems to increase the susceptibility to Leishmania, as there were no clinical signs or positive cultures from mice infected with Leishmania only.

L. infantum infection had little effect on the $\mathrm{Th}_{1} / \mathrm{Th}_{2}$ switch necessary for $P$. c. chabaudi control. The differences on IFN- $\gamma$ expression observed at day 14 in co-infected group were not sufficient to interfere with $P$. $c$. chabaudi parasitaemia as it was probably already under control. On the other hand, the presence of IFN- $\gamma$ at the time of Leishmania infection might have determined the high levels of IFN- $\gamma$ observed at day 56. Himmelrich et al. (2000) mention that exogenous IL-4 administration to C57BL/6 mice does not modify their resistant phenotype nor redirect effector cells to a $\mathrm{Th}_{2}$ phenotype. However, the presence of IL-4 at day 28, essential for P. c. chabaudi elimination, was probably a key factor on the outcome of Leishmania infection (IL-4 was not detected in Leishmania infected only) leading to an increase in the Leishmania parasite load at day 56 associated with a high IFN- $\gamma$ production. The higher expression levels of IL-4 on the day 28 might have been determinant, as it probably disturbed the fine tune of the mixed $\mathrm{Th}_{1} / \mathrm{Th}_{2}$ response observed in L. infantum infected mice (Honoré et al. 1998).

On the other hand, we can not exclude the importance 
of other cytokines during this concomitant parasitic infection. For example, a high expression and production of IL-10 as well as IFN- $\gamma$, were detected in dogs experimentally infected with $L$. infantum (Santos-Gomes et al. 2002) and by day 28 and 56 after infection with $L$. donovani, there was a high splenic and hepatic mRNA expression of IL-10 and TGF- $\beta$ in hamsters (Melby et al. 2001). Belkaid et al. (2002) also showed that the persistence of parasites of $L$. major in C57BL/6 is related with the production of IL- 10 by $\mathrm{CD} 4{ }^{+} \mathrm{CD} 25^{+}$regulatory $\mathrm{T}$ cells.

In conclusion, a fine tuning between $\mathrm{Th}_{1}$ and $\mathrm{Th}_{2}$ type responses is essential for the resolution of these parasitic infections. However the co-infection, that often occurs in nature, can disrupt this balance, leading to disease exacerbation.

Although this study comes across as a preliminary investigation, to our knowledge, is the first work in the murine model, that approaches immunological aspects in concomitant infection of $P$. c. chabaudi and L. infantum. Nevertheless, new research is currently undergoing in our laboratory, in order to explore and firmly define the impact of co-infection on $\mathrm{Th}_{1} / \mathrm{Th}_{2}$ immune responses.

\section{REFERENCES}

Belkaid Y, Piccirillo C, Mendez S, Shevach EM, Saks D 2002. $\mathrm{CD} 4{ }^{+} \mathrm{CD} 25^{+}$regulatory T cells control Leishmania major persistence and immunity. Nature 420: 502-507.

Boom R, Sol C, Salimans M, Jansen C, Dillen P, Nordaa J 1990. Rapid and simple method for purification of nucleic acids. J Clin Microbiol 28: 57-72.

Buffet PA, Sulahian A, Garin YJF, Nassar N, Derouin F 1995. Culture microtitration: a sensitive method for quantifying Leishmania infantum in tissues of infected mice. Antimicrob Agents Chemother 39: 2167-2168.

Carvalho E, Bacellar O, Brownell C, Regis T, Coffman R, Reed S 1994. Restoration of IFN-gamma production and lymphocyte proliferation in visceral leishmaniasis. J Immunol 152: 5949-5956.

Cenini P, Reeve AM, Neal RA 1989. Two new techniques for quantitative determination of Leishmania amastigotes. Trans $R$ Soc Trop Med Hyg 83: 194-195.

Centeno-Lima S, Silveira H, Casimiro C, Aguiar P, do Rosário VE 2002. Kinectics of cytokine expression in mice with invasive aspergillosis: lethal infection and protection. FEMS Immunol Med Microbiol 32: 167-173.

Coleman RE, Edman JD, Sempreviso LH 1988a. Leishmania mexicana: effect of concomitant malaria on cutaneous leishmaniasis. Development of lesions in a Leishmania-susceptible (BALB/c) strain of mouse. Exp Parasitol 65: 269-276.

Coleman RE, Edman JD, Semprevis, LH 1988b. Interactions between Plasmodium yoelii and Leishmania mexicana amazonensis in Leishmania resistant C57BL/6 mice. Am J Trop Med Hyg 39: 540-544.

Coleman RE, Edman JD, Sempreviso LH 1988c. Metastasis of Leishmania mexicana in Leishmania-resistant mouse strain (C/57) following concomitant malarial infection. Ann Trop Med Parasitol 82: 399-401.

Cortes S, Rolão N, Ramada J, Campino L 2004. PCR as a rapid and sensitive tool in the diagnosis of human and canine leishmaniasis using Leishmania donovani s.l. specific kinetoplastid primers. Trans R Soc Trop Med Hyg 98: 1217.

Gumy A, Louis JA, Launois P 2004. The murine model of infection with Leishmania major and its importance for the deciphering of mechanisms underlying differences in Th cells differentiation in mice from different genetic backgrounds. Int J Parasitol 34: 433-444.

Heinzel FP, Sadick MD, Holaday BJ, Coffman RL, Locksley RM 1989. Reciprocal expression of interferon- $\gamma$ or interleukin-4 during the resolution or progression of murine leishmaniasis: evidence for expansion of distinct helper Tcell subsets. J Exp Med 169: 59-72.

Himmelrich H, Launois P, Maillard I, Biedermann T, TacchiniCottier F, Locksley RM, Rocken M, Louis JA 2000. In $\mathrm{BALB} / \mathrm{c}$ mice, IL-4 production during the initial phase of infection with $L$. major is necessary and sufficient to instruct $\mathrm{Th}_{2}$ cell development resulting in progressive disease. J Immunol 164: 4819-4825.

Honoré S, Garin Y, Sulahian A, Gangneux JP Derouin F 1998. Influence of the host and parasite strain in a mouse model of visceral Leishmania infantum infection. FEMS Immunol Med Microbiol 21: 231-239.

Kaye PM, Curry AJ, Blackwell JM 1991. Differential production of $\mathrm{Th}_{1}$ and $\mathrm{Th}_{2}$-derived cytokines does not determine the genetically controlled or vaccine-induced rate of cure in murine visceral leishmaniasis. J Immunol 146: 2763-2770.

Langhorne $\mathrm{J}$ 1989. The role of $\mathrm{CD}_{4}{ }^{+} \mathrm{T}$-cells in the immune responses to Plasmodium chabaudi. Parasitol Today 5: 362-364.

Melby PC, Chandrasekar B, Zhao W, Coe JE 2001. The hamster as a model of human visceral leishmaniasis: progressive disease and impaired generation of nitric oxide in the face of prominent $\mathrm{Th}_{1}$-like cytokine response. J Immunol 166: 1912-1920.

Nandy A, Addy M, Guha SK, Maji AK, Chaudhuri D, Chatterjee P 1995. Co-existent Kala-azar and malaria in India. Trans $R$ Soc Trop Med Hyg 89: 516.

Reed S, Scott P (1993) T-cell and cytokine responses in leishmaniasis. Curr Opin Immunol 5: 524-531

Sah SP, Sharma SK, Rani S 2002. Kala-azar associated with malaria. Arch Pathol Lab Med 126: 382-383.

Santos-Gomes G, Rosa R, Leandro C, Cortes S, Romão P, Silveira H 2002. Cytokine expression during the outcome of canine experimental infection by L. infantum. Vet Immunol Immunopathol 88: 21-30.

Taylor-Robinson AW 1998. Immunoregulation of malarial infection: balancing the vices and virtues. Int J Parasitol 28: 135-48.

Taylor-Robinson AW, Phillips RS 1994. B cells are required for the switch from $\mathrm{Th}_{1}$ to $\mathrm{Th}_{2}$-regulated immune responses to Plasmodium chabaudi chabaudi infection. Infect Immun 62: 2490-2498.

Titus, R.G., Marchand, M., Boon, T., Louis, J.A. 1985. A limiting diluition assay for quantifying Leishmania major in tissues of infected mice. Parasite Immunol 7: 545.

Zhong S, Stevenson MM 2000. Central role of endogenous gamma interferon in protective immunity against bloodstage Plasmodium chabaudi AS infection. Infect Immun 68: 4399-4406. 